\title{
A contribution to infinite disjoint covering systems
}

\author{
par JÁNOs BARÁT et PÉTER P. VARJÚ
}

\begin{abstract}
RÉSUMÉ. Supposons que la famille de suites arithmétiques $\left\{d_{i} n+\right.$ $\left.b_{i}: n \in \mathbb{Z}\right\}_{i \in I}$ soit un recouvrement disjoint des nombres entiers. Nous prouvons qui si $d_{i}=p^{k} q^{l}$ pour des nombres premiers $p, q$ et des entiers $k, l \geq 0$, il existe alors un $j \neq i$ tel que $d_{i} \mid d_{j}$. On conjecture que le résultat de divisibilité est vrai quelques soient les raisons $d_{i}$.

Un recouvrement disjoint est appelé saturé si la somme des inverses des raisons est égale à 1 . La conjecture ci-dessus est vraie pour des recouvrements saturés avec des $d_{i}$ dont le produit des facteurs premiers n'est pas supérieur à 1254 .
\end{abstract}

ABStraCt. Let the collection of arithmetic sequences $\left\{d_{i} n+b_{i}\right.$ : $n \in \mathbb{Z}\}_{i \in I}$ be a disjoint covering system of the integers. We prove that if $d_{i}=p^{k} q^{l}$ for some primes $p, q$ and integers $k, l \geq 0$, then there is a $j \neq i$ such that $d_{i} \mid d_{j}$. We conjecture that the divisibility result holds for all moduli.

A disjoint covering system is called saturated if the sum of the reciprocals of the moduli is equal to 1 . The above conjecture holds for saturated systems with $d_{i}$ such that the product of its prime factors is at most 1254 .

A Beatty sequence is defined by $S(\alpha, \beta):=\{\lfloor\alpha n+\beta\rfloor\}_{n=1}^{\infty}$, where $\alpha$ is positive and $\beta$ is an arbitrary real constant. A conjecture of Fraenkel asserts that if $\left\{S\left(\alpha_{i}, \beta_{i}\right): i=1 \ldots m\right\}$ is a collection of $m \geq 3$ Beatty sequences which partitions the positive integers then $\alpha_{i} / \alpha_{j}$ is an integer for some $i \neq j$. Special cases of the conjecture were verified by Fraenkel [2], Graham [4] and Simpson [9]. For more references on Beatty sequences see [1] and [11]. If $\alpha$ is integral, then $S(\alpha, \beta)$ is an arithmetic sequence. Mirsky and Newman, and later independently Davenport and Rado proved that if $\left\{\left\{a_{i} n+b_{i}\right\}: i=1 \ldots m\right\}$ is a partition of the positive integers, then $a_{i}=a_{j}$ for some $i \neq j$. This settles Fraenkel's conjecture for integral $\alpha$ 's. We formulate a related conjecture for partitions to infinite number of arithmetic sequences.

We denote by $A(d, b)$ the arithmetic sequence $\{d n+b: n \in \mathbb{Z}\}$. Let a collection $\mathcal{S}$ of arithmetic sequences $\left\{A\left(d_{i}, b_{i}\right): i \in I\right\}$ be called a covering 
system (CS), if the union of the sequences is $\mathbb{Z}$. The CS is finite or infinite according to the set $I$. The numbers $d_{i}$ are called the moduli of the CS. A conjecture similar to Fraenkel's was posed by Schinzel, that for any finite $\mathrm{CS}$, there is a pair of distinct indices $i, j$ for which $d_{i} \mid d_{j}$. This was verified by Porubský [8] assuming some extra conditions.

When the sequences of a CS are disjoint, it is called a disjoint covering system (DCS). The structure of DCS's is a wide topic of research. We only mention here a few results about IIDCS's (such DCS's that the number of sequences are infinite, and the moduli are distinct). For further references see [7]. There is a natural method to construct DCS's, the following construction appeared in [10]:

Example 1. Let $I=\mathbb{N}$, and $d_{1}\left|d_{2}\right| d_{3} \ldots$ be positive integers. Define the $b_{i}$ 's recursively to be an integer of minimal absolute value not covered by the sequences $A\left(d_{j}, b_{j}\right),(j<i)$.

Indeed this gives a DCS; if $A\left(d_{i}, b_{i}\right)$ and $A\left(d_{j}, b_{j}\right)(j<i)$ do intersect then $A\left(d_{i}, b_{i}\right) \subset A\left(d_{j}, b_{j}\right)$ as $d_{j} \mid d_{i}$, which contradicts the definition of $b_{i}$. Also the definition of $b_{i}$ guarantees that an integer of absolute value $n$ is covered by one of the first $2 n+1$ sequences.

If the sum of the reciprocals of the moduli equals 1 , we call the DCS saturated. Apparently every finite DCS is saturated, but this property is rather "rare" for IIDCS's. The IIDCS in the example above is saturated only for $d_{i}=2^{i}$. Stein [10] asked whether this is the unique example. Krukenberg [5] answered this in the negative, then Fraenkel and Simpson [3] characterised all IIDCS, whose moduli are of form $2^{k} 3^{l}$. Lewis [6] proved that if a prime greater than 3 divides one of the moduli, then the set of all prime divisors of the moduli is infinite.

We formulate the following conjecture:

Conjecture 2. If $\left\{A\left(d_{i}, b_{i}\right): i \in I\right\}$ is a DCS, then for all $i$ there exists an index $j \neq i$ such that $d_{i} \mid d_{j}$.

This conjecture is valid for the above examples, and also valid for those appearing in [3]. It is also known for finite DCS's, being a consequence of Corollary 2 of [8].

We prove the following special case of Conjecture 2 .

Theorem 3. If $\left\{A\left(d_{i}, b_{i}\right): i \in I\right\}$ is a DCS, and $d_{i}=p^{k} q^{l}$ for some primes $p, q$ and integers $k, l \geq 0$, then $d_{i} \mid d_{j}$ for some $j \neq i$.

Proof. Let $(a, b)$ denote the greatest common divisor of the integers $a, b$, and $[a, b]$ their least common multiple. We will use the fact, that the sequences $A\left(d_{1}, b_{1}\right)$ and $A\left(d_{2}, b_{2}\right)$ are disjoint if and only if $\left(d_{1}, d_{2}\right) \nmid x_{1}-x_{2}$, where $x_{i}$ is an arbitrary number covered by $A\left(d_{i}, b_{i}\right)$ for $i=1,2$. 
If $l=0$, consider the sequence $A\left(d_{j}, b_{j}\right)$ that covers $b_{i}+p^{k-1}$. Then $\left(d_{j}, d_{i}\right) \nmid b_{i}+p^{k-1}-b_{i}=p^{k-1}$. Thus $\left(d_{j}, d_{i}\right)=p^{k}=d_{i}$, so $d_{i} \mid d_{j}$ which was to be proved. Now we may assume that $k, l>0$.

Assume to the contrary, that the theorem is false. Defining $b_{j}^{\prime}=b_{j}-b_{i}$, we get another DCS $\left\{A\left(d_{j}, b_{j}^{\prime}\right): j \in I\right\}$, where $b_{i}^{\prime}=0$. Hence we may assume that $b_{i}=0$.

Let $A_{j}=A\left(d_{j}, b_{j}\right) \cap A\left(p^{k-1} q^{l-1}, 0\right)$. Either $A_{j}$ is empty or an arithmetic sequence, whose modulus is $\left[p^{k-1} q^{l-1}, d_{j}\right]$. Let $B_{j}=\left\{\frac{x}{p^{k-1} q^{l-1}} \mid x \in A_{j}\right\}$. The nonempty sequences among the $B_{j}$ 's form a DCS. Notice that $p q$ divides the modulus of $B_{j}$ if and only if $d_{i}=p^{k} q^{l} \mid d_{j}$. Since the modulus of $B_{i}$ is $p q$, it remains to prove the theorem for $k=l=1$.

Assume $d_{i}=p q$, and $p q \nmid d_{j}$ for $i \neq j$. Assume that $p+q$ is covered by the sequence $A\left(d_{t}, b_{t}\right)$ of the DCS. We prove that $p \nmid d_{t}$.

Assume to the contrary that $p \mid d_{t}$. Let $d_{t}=d \cdot p^{m}$, where $p \nmid d$. Then $(p q, d) \mid q$, and there exist a pair of positive integers $u, v$ such that $q=$ $p q \cdot u-d \cdot v$. Let $a=p+q+d v=p+p q u$. Assume that $a$ is covered by $A\left(d_{s}, b_{s}\right)$. If $A\left(d_{s}, b_{s}\right)$ and $A\left(d_{t}, b_{t}\right)$ are the same sequences, then $d_{s}=d_{t}$, and $p \mid d_{s}$. Otherwise $\left(d_{s}, d_{t}\right) \nmid p+q+d v-p-q=d v$, which yields $p \mid d_{s}$. Since $p q \nmid a, s \neq i$, and $\left(d_{s}, d_{i}\right) \nmid p+p q u-p q u=p$, thus $q \mid d_{s}$. This contradicts $d_{i} \nmid d_{s}$.

Similar argument shows $q \nmid d_{t}$, which contradicts $\left(d_{i}, d_{t}\right) \nmid b_{i}-b_{t}$. So the proof is complete.

As a byproduct of the previous proof, we got the following lemma:

Lemma 4. Suppose there is a DCS $\left\{A\left(d_{i}, b_{i}\right): i \in I\right\}$ and an index $i \in I$ such, that $d_{i}=p_{1}^{\alpha_{1}} p_{2}^{\alpha_{2}} \ldots p_{k}^{\alpha_{k}}$, where the $p$ 's are distinct primes, and $d_{i} \nmid d_{j}$ for all $j \neq i$. Then there exists another DCS $\left\{A\left(\hat{d}_{i}, \hat{b}_{i}\right): i \in \hat{I}\right\}$ and an index $\hat{i} \in \hat{I}$ such that $\hat{d}_{\hat{i}}=p_{1} p_{2} \ldots p_{k}$ and $\hat{d}_{\hat{i}} \nmid \hat{d}_{j}$ for all $j \neq \hat{i}$.

So it is sufficient to verify the conjecture for square-free moduli. When $d_{i}$ has more than two different prime factors, the situation seems to be much more complicated. We can still say something for saturated DCS's. We need the following concepts:

Suppose $A \subseteq \mathbb{Z}$. Let $S_{n}(A)=|\{x \in A:-n<x<n\}|$ be the number of elements of $A$ with absolute value less than $n$. We define the density of $A$ to be $d(A)=\lim _{n \rightarrow \infty} \frac{S_{n}(A)}{2 n-1}$ if the limit exists, and in that case we say, that the density of $A$ exists. We will use following facts. The density is finitely additive, and the density of arithmetic sequences exist, and $d(A(d, b))=\frac{1}{d}$. Let $\left\{A\left(d_{i}, b_{i}\right): i \in I\right\}$ be a saturated DCS, and $J \subseteq I$. Lemma 2.2 of $[6]$ states, that the density of $X:=\bigcup_{j \in J} A\left(d_{j}, b_{j}\right)$ exists, and $d(X)=\sum_{j \in J} \frac{1}{d_{j}}$. 
Let $a$ be an arbitrary and $b$ a positive integer. Denote by $a \bmod b$ the unique integer $0 \leq c<b$, that $b \mid a-c$.

Lemma 5. Suppose there is a saturated DCS $\left\{A\left(d_{i}, b_{i}\right): i \in I\right\}$, and an index $i \in I$ such that for all $j \neq i d_{i} \nmid d_{j}$. Let $D$ denote the set of positive divisors of $d_{i}$ different from 1 and $d_{i}$. Then there exist nonnegative real numbers $x_{s, t}$, where $s \in D$ and $0 \leq t<s$, such that for all $0<u<d_{i}$

$$
\sum_{s \in D} x_{s, u \bmod s}=1
$$

and $x_{s, 0}=0$ for all $s \in D$.

Proof. Assume $b_{i}=0$. Denote by $I_{s, t}$ the set of indices $j \neq i$ such that $\left(d_{i}, d_{j}\right)=s$, and $b_{j} \bmod s=t$. Notice that $I_{s, 0}=\emptyset$ and $I=$ $\{i\} \cup \bigcup_{s \in D ; 0 \leq t<s} I_{s, t}$. Let $y_{s, t}=d\left(\bigcup_{j \in I_{s, t}} A\left(d_{j}, b_{j}\right)\right)$. Then $y_{s, 0}=0$ for all $s$. Since the DCS is saturated, $y_{s, t}=\sum_{j \in I_{s, t}} \frac{1}{d_{j}}$.

Let $0<u<d_{i}$ and

$$
Y_{s, u}=\bigcup_{j \in I_{s, u \bmod s}}\left(A\left(d_{j}, b_{j}\right) \cap A\left(d_{i}, u\right)\right) .
$$

If some element of $A\left(d_{i}, u\right)$ is covered by the sequence $A\left(d_{j}, b_{j}\right)$, and $\left(d_{i}, d_{j}\right)=s$, then $s \mid u-b_{j}$. Thus $A\left(d_{i}, u\right)=\bigcup_{s \in D} Y_{s, u}$. Notice that $Y_{s, u}$ is the union of sequences of form $A\left(d_{j} \cdot \frac{d_{i}}{s}, u+k d_{i}\right)$ for some $k$, depending on $j$. Consider the saturated DCS, that consists of the sequences $A\left(d_{j}, b_{j}\right) \cap A\left(d_{i}, u\right)$, for $j \in I, 0 \leq u<d_{i}$. As $Y_{s, u}$ is the union of some sequences of this DCS:

$$
d\left(Y_{s, u}\right)=\frac{s}{d_{i}} \sum_{j \in I_{s, u \bmod s}} \frac{1}{d_{j}}=\frac{s}{d_{i}} y_{s, u \bmod s} .
$$

Since $A\left(d_{i}, u\right)=\bigcup_{s \in D} Y_{s, u}, d\left(d_{i}, u\right)=\sum_{s \in D} d\left(Y_{s, u}\right)$, we get $\frac{1}{d_{i}}=\sum_{s \in D} \frac{s}{d_{i}} y_{s, u \bmod s}$. We finish the proof by setting $x_{s, t}=\frac{y_{s, t}}{s}$.

We conjecture, that the system of linear equations in Lemma. 5 has no solutions, which would prove Conjecture 2 for saturated DCS's. We have checked this with a computer program for square-free numbers $d_{i} \leq 1254=$ $2 \cdot 3 \cdot 11 \cdot 19$.

\section{Acknowledgements}

The authors gratefully thank the referee for her or his useful comments, and Anna Pósfai for the french translation of the abstract. This research has been supported by a Hungarian National Grant, OTKA T.34475. 


\section{References}

[1] J. BARÁt, P.P. VARJú, Partitioning the positive integers to seven Beatty sequences. Indag. Math. 14 (2003), 149-161.

[2] A.S. Fraenkel, Complementing and exactly covering sequences. J. Combin. Theory Ser. A 14 (1973), 8-20.

[3] A.S. Fraenkel, R.J. Simpson, On infinite disjoint covering systems. Proc. Amer. Math. Soc. 119 (1993), 5-9.

[4] R.L. Graham, Covering the positive integers by disjoint sets of the form $\{[n \alpha+\beta]$ : $n=1,2, \ldots\}$. J. Combin Theory Ser. A 15 (1973), 354-358.

[5] C.E. Krukenberg, Covering sets of the integers. Ph. D. Thesis, Univ. of Illinois, UrbanaChampaign, IL, (1971)

[6] E. LEWIS, Infinite covering systems of congruences which don't exist. Proc. Amer. Math. Soc. 124 (1996), 355-360.

[7] Š. Porubský, J. Schönheim, Covering Systems of Paul Erdős, Past, Present and Future. In Paul Erdős and his Mathematics I., Springer, Budapest, (2002), 581-627.

[8] Š. PoRUBSKÝ, Covering systems and generating functions. Acta Arithm. 26 (1975), 223231.

[9] R.J. SIMPSON, Disjoint covering systems of rational Beatty sequences. Discrete Math. 92 (1991), 361-369.

[10] S.K. Stein, Unions of Arithmetic Sequences. Math. Annalen 134 (1958), 289-294.

[11] R. Tijdeman, Fraenkel's conjecture for six sequences. Discrete Math. 222 (2000), 223-234.

\section{János BARÁt}

Bolyai Institute

University of Szeged

Aradi vértanúk tere 1 .

Szeged, 6720 Hungary

E-mail : jbarat@math.u-szeged.hu

Péter VARJú

Bolyai Institute

University of Szeged

Aradi vértanúk tere 1 .

Szeged, 6720 Hungary

E-mail : Varju.Peter.Pal@stud.u-szeged.hu 